\title{
Risk stratification in motor area-related glioma surgery based on navigated transcranial magnetic stimulation data
}

\author{
Tizian Rosenstock, ${ }^{1}$ Ulrike Grittner, PhD, ${ }^{2}$ Güliz Acker, MD, ${ }^{1}$ Vera Schwarzer, ${ }^{1}$ Nataliia Kulchytska, ${ }^{1}$ \\ Peter Vajkoczy, PhD, ${ }^{1}$ and Thomas Picht, MD ${ }^{1}$
}

Departments of ${ }^{1}$ Neurosurgery and ${ }^{2}$ Biostatistics and Clinical Epidemiology, Charité University Medicine, Berlin, Germany

OBJECTIVE Navigated transcranial magnetic stimulation (nTMS) is a noninvasive method for preoperatively localizing functional areas in patients with tumors in presumed motor eloquent areas. The aim of this study was to establish an nTMS-based risk stratification model by examining whether the results of nTMS mapping and its neurophysiological data predict postoperative motor outcome in glioma surgery.

METHODS Included in this study were prospectively collected data for 113 patients undergoing bihemispheric nTMS examination prior to surgery for gliomas in presumed motor eloquent locations. Multiple ordinal logistic regression analysis was performed to test for any association between preoperative nTMS-related variables and postoperative motor outcome.

RESULTS A new motor deficit or deterioration due to a preexisting deficit was observed in $20 \%$ of cases after 7 days and in $22 \%$ after 3 months. In terms of tumor location, no new permanent deficit was observed when the distance between tumor and corticospinal tract was greater than $8 \mathrm{~mm}$ and the precentral gyrus was not infiltrated $(p=0.014)$. New postoperative deficits on Day 7 were associated with a pathological excitability of the motor cortices (interhemispheric resting motor threshold [RMT] ratio $<90 \%$ or $>110 \%, p=0.031$ ). Interestingly, motor function never improved when the RMT was significantly higher in the tumorous hemisphere than in the healthy hemisphere (RMT ratio > 110\%).

CONCLUSIONS The proposed risk stratification model, based on objective functional-anatomical and neurophysiological measures, enables one to counsel patients about the risk of functional deterioration or the potential for recovery. https://thejns.org/doi/abs/10.3171/2016.4.JNS152896

KEY WORDS navigated transcranial magnetic stimulation; brain tumor surgery; glioma; predictive model; motor outcome; diffusion tensor imaging; oncology; surgical technique

$\mathrm{I}$ $\mathrm{T}$ is widely accepted that extensive resection of malignant gliomas can improve patient survival and quality of life. ${ }^{1,12,18,31}$ Yet this goal must be balanced against the risk of surgically induced neurological deficits, especially when planning surgery in presumed motor eloquent areas. ${ }^{21,29}$ Whereas permanent motor deficits clearly affect a patient's well-being, even transient deficits can have devastating effects, especially in elderly patients, by preventing the timely administration of adjuvant therapy or by causing complications. ${ }^{21}$

Intraoperative neurophysiological monitoring (IOM) such as direct cortical and subcortical stimulation has

ABBREVIATIONS CST = corticospinal tract; DTI = diffusion tensor imaging; EMG = electromyography; FAT = fractional anisotropy threshold; FDI = first dorsal interosseous; GTR = gross-total resection; IntCaps $=\leq 8 \mathrm{~mm}$ from CST; IOM = intraoperative neurophysiological monitoring; KPS = Karnofsky Performance Scale; M0 = neither infiltrating nor adjacent to primary motor cortex; $\mathrm{M} 1=$ infiltration of primary motor cortex or corticospinal tract; M2 = directly adjacent to M1; MEP = motor evoked potential; MRC = Medical Research Council; $n T M S=$ navigated transcranial magnetic stimulation; PR = partial resection; RMT = resting motor threshold; STR = subtotal resection SUBMITTED December 21, 2015. ACCEPTED April 6, 2016.

INCLUDE WHEN CITING Published online June 3, 2016; DOI: 10.3171/2016.4.JNS152896. 
been established to increase safety during the resection of rolandic brain tumors. ${ }^{7,13}$ Yet even when IOM is routinely available, preoperative risk assessment is essential to allow for optimal patient counseling and treatment planning. Navigated transcranial magnetic stimulation (nTMS) has been extensively validated as a reliable tool for exactly analyzing the spatial relation between brain tumors and primary motor areas in a noninvasive fashion. ${ }^{17,23,24,32}$ It has been shown that preoperatively examining patients with nTMS enables more extensive resections while reducing the rate of functional deficits. ${ }^{10,16,17,25}$ In addition to the topographical information offered, the nTMS examination also provides neurophysiological parameters whose clinical relevance has not been elucidated as yet. ${ }^{26}$

The aim of this study was to analyze whether the topographical and neurophysiological measurements provided by nTMS can be used to predict the motor outcome in patients scheduled for surgery of malignant gliomas near motor eloquent areas.

\section{Methods \\ Ethical Standard}

The study protocol accords with the ethical standards of the Declaration of Helsinki and was approved by the Ethics Commission of the Charité University Hospital. All patients provided written informed consent for medical evaluations and treatments within the scope of the study.

\section{Patient Sample}

Prospective data collection started in October 2007 and ended in December 2014. Patients presenting with gliomas, judged according to anatomical MRI to 1) compress or infiltrate the motor cortex and/or 2) be closely related to the corticospinal tract (CST), were included in this study to evaluate the possibility of resection by the responsible senior neurosurgeon. Exclusion criteria for an nTMS evaluation were frequent generalized seizures (more than 1 per week) or cranial implants. In each case a detailed neurological examination was performed preoperatively and 7 days and 3 months postoperatively. All findings were saved in a custom-made database. The following biographical and clinical items were documented: age, sex, antiepileptic or antiedematous medication, Karnofsky Performance Scale (KPS) ${ }^{30}$ motor status (according to the British Medical Research Council [MRC] grade where 0 means no muscle activation and 5 means normal muscle strength), ${ }^{20}$ and duration of symptoms. The Department of Pathology determined tumor histology according to the WHO classification. ${ }^{19}$

\section{Magnetic Resonance Imaging}

Cerebral MRI with a contrast-enhanced 3D gradient echo sequence, fluid-attenuated inversion recovery (FLAIR) sequence, and diffusion tensor imaging (DTI) sequence was performed using a 1.5- or 3-T MR imaging unit (GE Healthcare) with an 8-channel head coil, as previously described in detail. ${ }^{11}$ An interdisciplinary team of neurosurgeons and neuroradiologists interpreted all MR scans. The contrast-enhanced 3D gradient echo sequence was imported into the nTMS system (eXimia, Nexstim Oy) for generating a $3 \mathrm{D}$ reconstruction, which allows for controlling the spatial relation between the brain and the stimulation location.

\section{Navigated TMS}

Both hemispheres were examined using nTMS, as specified previously. ${ }^{24}$ In short, TMS relies on the principle of electromagnetic induction. A powerful electrical current is rapidly discharged through a figure-8 TMS coil, generating a brief, cone-shaped magnetic field. This magnetic field penetrates the skull unattenuated and induces an electrical field in the underlying brain. Stimulation of pyramidal cells, their axons, or surrounding interneurons may result in a motor evoked potential (MEP) depending on the stimulation location and intensity.,23 Motor evoked potentials are recorded by the system's integrated electromyography (EMG) unit using surface electrodes (sampling rate $3 \mathrm{kHz}$, resolution $0.3 \mathrm{mV}$; Neuroline 720 , Ambu). Depending on the requirements of each case as regards tumor location and individual clinical situation, EMG activity from the following muscles was recorded: abductor pollicis brevis, first dorsal interosseous (FDI), adductor digiti minimi muscles for the upper extremity and the tibialis anterior and abductor hallucis brevis muscles for the lower extremity.

During the nTMS examination, patients were asked to relax and to keep their eyes open. First, the "hot spot" 27 of the FDI muscle was identified by applying TMS in a dense raster and different coil rotations to obtain the best topographic accuracy. ${ }^{25}$ Then, the resting motor threshold (RMT), defined as the lowest stimulation intensity sufficient to induce an MEP $(\geq 50 \mu \mathrm{V})$ in at least 5 of 10 stimulations, was determined and reported in volts per meter at the top of the cortex for each hemisphere. Subsequently, peritumoral mapping for the upper (stimulation intensity: $110 \%$ RMT) and lower (median stimulation intensity: $130 \%$ RMT) extremity was performed..$^{18}$ Finally, mapping with high specificity (stimulation intensity: 105\% RMT) was performed to specifically outline the primary motor cortex along the precentral gyrus. The MEP-positive stimulation locations from this sequence were used for subsequent surgical planning.

The nTMS examination and planning process are visualized in Fig. 1.

\section{Surgical Planning}

For surgical planning, the TMS stimuli locations outlining the primary motor cortex were imported into the surgical planning software in the DICOM format (iPlan 2.0, BrainLab). For DTI tracking, the TMS stimulation points were enlarged to a radius of $3 \mathrm{~mm}$ to generate a continuous seed point area. ${ }^{11}$ To improve tracking robustness, a second seed point was placed into the anterolateral portion of the ipsilateral cerebral peduncle. ${ }^{6}$ Afterward, fiber tracking at $75 \%$ of the fractional anisotropy threshold (FAT) and minimum fiber length of $110 \mathrm{~mm}$ was performed, as described in detail elsewhere. ${ }^{11}$ Clearly aberrant tracts were removed, and the minimum distance between the tumor and the CST was measured. Finally, the 

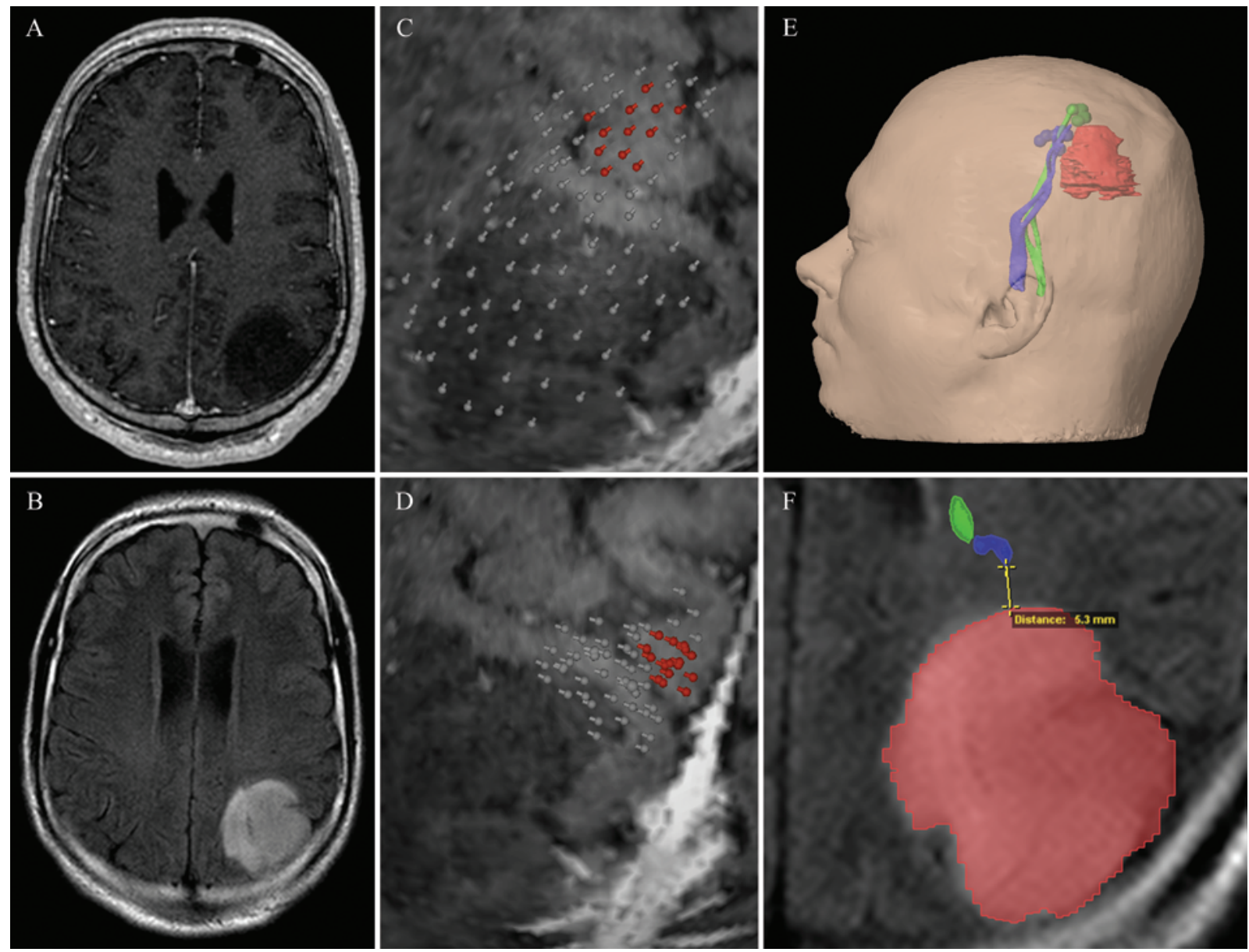

FIG. 1. Illustration of our nTMS workflow. In this case, the examined patient had a non-gadolinum-enhancing (post)central tumor on T1-weighted (A) and FLAIR (B) MRI sequences. Cortical area maps for the upper (C) and lower (D) extremities. The spatial relation between the tumor and the CST (upper extremities, blue; lower extremities, green; tumor, red) is visualized (E) and its minimal distance is measured (F) using the FLAIR sequence for this nonenhancing low-grade glioma. Figure is available in color online only.

tumorous tissue was visualized in red, and the final map consisting of segmented tumor, TMS stimulation points outlining the primary motor cortex, and TMS-based fiber tracts was made available to the surgical team via the hospital's intranet (Fig. 1).

\section{Surgery}

All patients underwent surgery for tumor resection. The surgical strategy was decided based on each patient's case history, clinical findings, MRI results, functional map provided by TMS mapping, and TMS-based DTI fiber tracking. During surgery, the functional data were made available either on the navigational screen or by projection into the microscopic view. Intraoperative neurophysiological mapping and monitoring were used at the surgeon's discretion. If applied, a standardized procedure consisting of monopolar anodal trains of 5 square-wave pulses $(0.3 \mathrm{msec}, 400 \mathrm{~Hz})$ for cortical and subcortical mapping as well as monitoring of motor function was applied, as previously described. ${ }^{10,14}$ The following signs were regarded as IOM stop criteria: persistent MEP amplitude reduction over 50\% and reproducible MEPs at $5 \mathrm{~mA}$ during sub- cortical dissection. Yet, termination of the tumor resection was decided for each individual based on the specific surgical circumstances and IOM phenomena.

\section{Extent of Resection}

Nearly all patients (96\%) underwent MRI within 48 hours after surgery. Extent of resection was analyzed critically by an independent neuroradiologist and determined by volumetric assessment of the residual tumor volume based on gadolinium-enhanced T1-weighted images or on FLAIR images in low-grade gliomas. To assess residual tumor volume, we divided resection results into 4 groups according to a common and widely used classification system: gross-total resection (GTR; no residual contrast-enhancing tissue on T1-weighted images and no residual hyperintense tissue on FLAIR images of nonenhancing tumors), subtotal resection (STR; residue $<10 \mathrm{~cm}^{3}$ ), partial resection (PR; residue $>10 \mathrm{~cm}^{3}$ ), and biopsy. ${ }^{4}$

\section{Statistical Analysis}

Descriptive statistics were used to analyze the patient 
TABLE 1. Univariate analyses of patient characteristics at baseline, according to postoperative motor status

\begin{tabular}{|c|c|c|c|c|c|c|c|c|c|c|}
\hline \multirow[b]{2}{*}{ Variable } & \multirow{2}{*}{$\begin{array}{l}\text { No. of } \\
\text { Patients }\end{array}$} & \multicolumn{4}{|c|}{ MRC Grade at 7 Days Postop } & \multirow{2}{*}{$\begin{array}{c}\text { No. of } \\
\text { Patients }\end{array}$} & \multicolumn{4}{|c|}{ MRC Grade at 3 Mos Postop } \\
\hline & & Mean & Median & IQR & $p$ Value & & Mean & Median & IQR & $\mathrm{p}$ Value \\
\hline Sex & & & & & $0.735^{*}$ & & & & & $0.566^{*}$ \\
\hline Male & $58(51 \%)$ & 4.0 & 4.0 & $4.0-5.0$ & & $44(51 \%)$ & 3.9 & 4.0 & $3.25-5.0$ & \\
\hline Female & $55(49 \%)$ & 4.1 & 4.0 & $4.0-5.0$ & & $43(49 \%)$ & 4.1 & 4.0 & $4.0-5.0$ & \\
\hline Preop motor status & & & & & $<0.001 \dagger$ & & & & & $0.001 \dagger$ \\
\hline MRC grade $\leq 3$ & $12(11 \%)$ & 2.5 & 3.0 & $2.25-3.0$ & & $9(10 \%)$ & 3.1 & 3.0 & $2.5-4.0$ & \\
\hline MRC grade 4 & $41(36 \%)$ & 4.0 & 4.0 & $4.0-4.0$ & & $32(37 \%)$ & 3.7 & 4.0 & $3.0-4.0$ & \\
\hline MRC grade 5 & $60(53 \%)$ & 4.4 & 5.0 & $4.0-5.0$ & & $46(53 \%)$ & 4.4 & 5.0 & $4.0-5.0$ & \\
\hline KPS score & & & & & $0.007 \dagger$ & & & & & $0.045 \dagger$ \\
\hline$\leq 70 \%$ & $17(15 \%)$ & 3.3 & 4.0 & $3.0-4.0$ & & $10(11 \%)$ & 3.5 & 4.0 & $3.0-4.0$ & \\
\hline $80 \%$ & $24(21 \%)$ & 3.8 & 4.0 & $3.0-5.0$ & & $20(23 \%)$ & 3.6 & 4.0 & $3.0-5.0$ & \\
\hline $90 \%$ & $44(39 \%)$ & 4.5 & 5.0 & $4.0-5.0$ & & $34(39 \%)$ & 4.3 & 4.5 & $4.0-5.0$ & \\
\hline $100 \%$ & $28(25 \%)$ & 4.1 & 5.0 & $4.0-5.0$ & & $23(26 \%)$ & 4.2 & 5.0 & $4.0-5.0$ & \\
\hline DOS $\ddagger$ & & & & & $0.017 \dagger$ & & & & & $0.003 \dagger$ \\
\hline no deficit & $58(53 \%)$ & 4.4 & 5.0 & $4.0-5.0$ & & $45(53 \%)$ & 4.4 & 5.0 & $4.0-5.0$ & \\
\hline$<4$ wks & $34(31 \%)$ & 3.5 & 4.0 & $3.0-4.0$ & & $27(32 \%)$ & 3.7 & 4.0 & $3.0-4.0$ & \\
\hline 4-12 wks & $8(7 \%)$ & 4.0 & 4.0 & $3.25-4.75$ & & $6(7 \%)$ & 3.7 & 4.0 & $2.75-4.25$ & \\
\hline$>12$ wks & $9(8 \%)$ & 3.8 & 4.0 & $3.0-4.0$ & & $7(8 \%)$ & 3.1 & 3.0 & $3.0-3.0$ & \\
\hline Affected hemisphere & & & & & $0.963^{*}$ & & & & & $0.071^{*}$ \\
\hline $\mathrm{Rt}$ & $57(50 \%)$ & 4.1 & 4.0 & $4.0-5.0$ & & $40(46 \%)$ & 3.8 & 4.0 & $3.0-5.0$ & \\
\hline $\mathrm{Lt}$ & $56(50 \%)$ & 4.0 & 4.0 & $4.0-5.0$ & & $47(54 \%)$ & 4.2 & 5.0 & $4.0-5.0$ & \\
\hline $\begin{array}{l}\text { nTMS-based tumor } \\
\text { localization } \S\end{array}$ & & & & & $0.010 \dagger$ & & & & & $0.139 \dagger$ \\
\hline M1 & $21(39 \%)$ & 3.4 & 4.0 & $2.5-5.0$ & & $16(38 \%)$ & 3.7 & 4.0 & $3.0-5.0$ & \\
\hline IntCaps & $17(31 \%)$ & 3.7 & 4.0 & $3.0-5.0$ & & $14(33 \%)$ & 3.5 & 4.0 & $2.75-5.0$ & \\
\hline M2 & $6(11 \%)$ & 4.5 & 4.5 & $4.0-5.0$ & & $5(12 \%)$ & 4.6 & 5.0 & $4.0-5.0$ & \\
\hline MO & $10(18 \%)$ & 4.7 & 5.0 & $4.0-5.0$ & & $7(17 \%)$ & 4.4 & 4.0 & $4.0-5.0$ & \\
\hline Tumor histology & & & & & $0.672 \pi$ & & & & & $0.825 \pi$ \\
\hline LGG & $17(15 \%)$ & 4.1 & 4.0 & $4.0-5.0$ & & $14(16 \%)$ & 3.9 & 4.0 & $3.0-5.0$ & \\
\hline HGG & $96(85 \%)$ & 4.1 & 4.9 & $4.0-5.0$ & & $73(84 \%)$ & 4.0 & 4.0 & $4.0-5.0$ & \\
\hline RMT ratio & & & & & $0.792 \dagger$ & & & & & $0.968 \dagger$ \\
\hline$<90 \%$ & $36(32 \%)$ & 4.1 & 4.0 & $4.0-5.0$ & & $26(30 \%)$ & 4.2 & 4.5 & $4.0-5.0$ & \\
\hline $90 \%-110 \%$ & $38(34 \%)$ & 4.1 & 4.0 & $4.0-5.0$ & & $31(36 \%)$ & 3.9 & 4.0 & $3.0-5.0$ & \\
\hline$>110 \%$ & $39(34 \%)$ & 3.9 & 4.0 & $3.0-5.0$ & & $30(34 \%)$ & 3.9 & 4.0 & $3.0-5.0$ & \\
\hline
\end{tabular}

DOS = duration of motor symptoms; HGG = high-grade glioma; IQR = interquartile range; LGG = low-grade glioma; RMT ratio = ratio of RMT value of affected hemi-

sphere/healthy hemisphere.

* Mann-Whitney U-test.

$\dagger$ Linear trend test (using Monte Carlo simulations for precision).

$\ddagger$ Four patients at 7 days and 2 patients at 3 months were unable to explain their medical histories appropriately; thus, they were excluded from analysis.

$\S$ Based on the number of patients with DTI: 54 patients at 7 days after surgery and 42 patients at 3 months after surgery.

If Fisher's exact test (using Monte Carlo simulations for precision).

data. It has been recently shown that neurophysiological values - in particular, the RMT-differ significantly between hemispheres in patients with brain tumor. ${ }^{26}$ Therefore, we calculated a ratio of the value of the affected hemisphere to that of the healthy hemisphere as an intraindividual measure (that is, the RMT ratio is calculated by dividing the value of the affected hemisphere by the value of the healthy hemisphere). For analyzing the association between the different variables and the postoperative motor status, we used the Mann-Whitney U-test, a linear trend test, and Fisher's exact test. In addition, we applied Monte Carlo simulations for greater precision.
To test the association of different characteristics with a change in motor status, we used general ordinal regression models, which allows deviation from the proportional odds assumption required in other ordinal regression models (Stata gologit2). Finally, we tested variables with a significant association in the univariate analyses in a multiple general ordinal regression model for testing a change in motor status at both 7 days and 3 months after surgery. The level of significance was 0.05 (2 sided). No adjustment for multiple testing was applied. Data analysis was performed using SPSS (IBM SPSS Statistics version 22, IBM Corp.) and Stata 13 (Stata IC, StataCorp). 

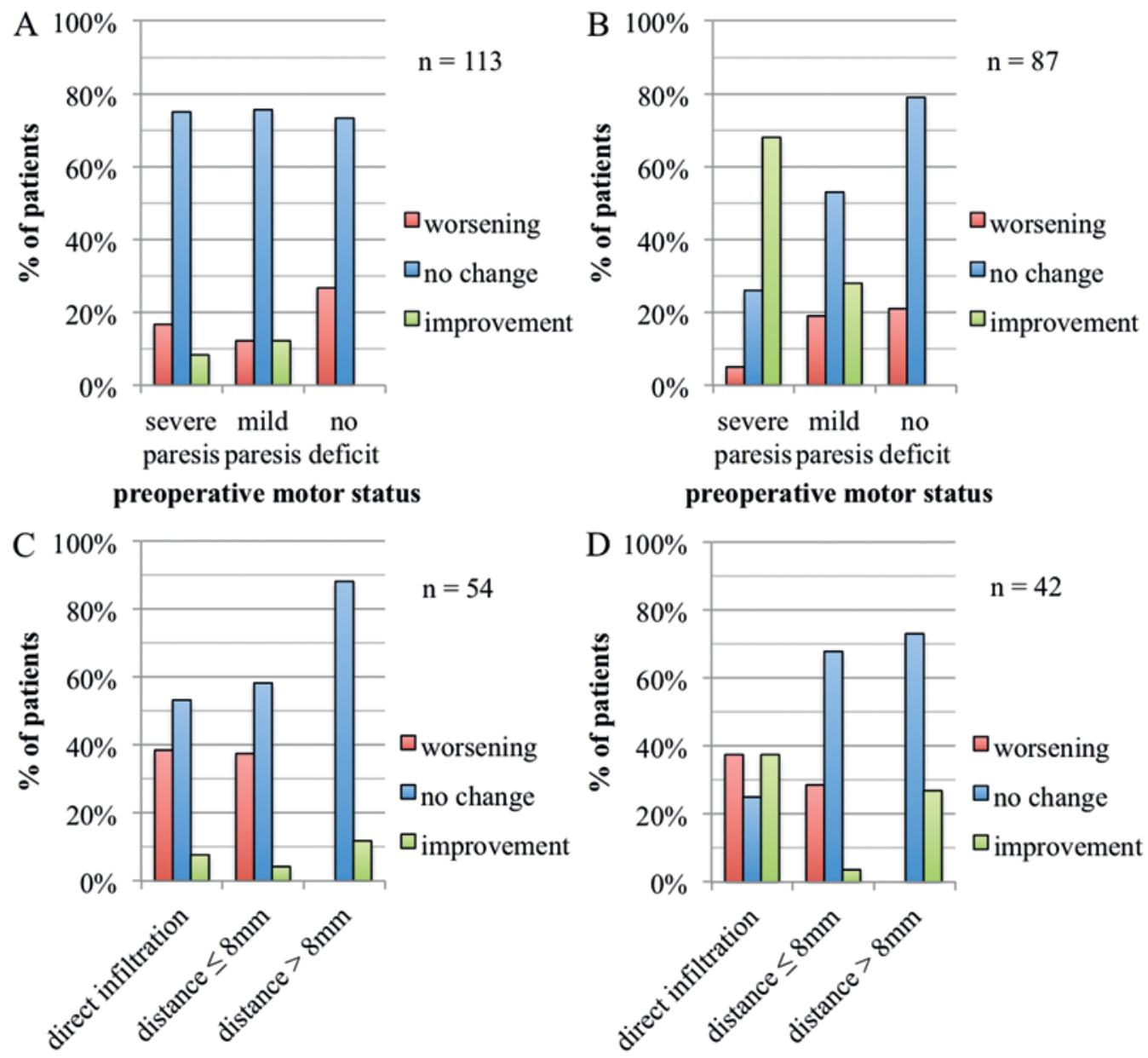

FIG. 2. Bar charts comparing the number of patients with improved, unchanged, and worse postoperative motor status after 7 days and after 3 months, according to the preoperative motor status ( $\mathbf{A}$ and $\mathbf{B}$, respectively) and according to the distance between the nTMS-based fiber tracts and suspected tumor tissue (C and D, respectively). Initially, 113 patients $(A)$ were examined, and 54 of them (C) underwent DTI. At 3 months after surgery, there were 87 patients (26 lost to follow-up, B), and 42 of them (D) underwent DTI. We did not observe any transient or permanent new deficits when the distance was greater than $8 \mathrm{~mm}$. Preoperative motor status was the independent variable in these subgroup analyses. $n=$ number of patients. Figure is available in color online only.

\section{Results}

\section{Patient Sample}

One hundred thirteen patients (55 female, 58 male) with a median age of 51 years (range 20-82 years) and a median KPS score of $90 \%$ (range 40\%-100\%) were included in this study; 53 of the patients $(47 \%)$ had a preoperative motor deficit. Both hemispheres were equally affected by tumor ( 57 right and 56 left hemisphere). About one-half (60 of 113) of the patients were preoperatively treated with antiepileptic drugs and $15.9 \%$ (18 of 113) with steroids.

Patient clinical characteristics and their association with postoperative motor status appear in Table 1. Motor function deteriorated after the operation in 1 (20\%) of 5 patients overall and improved in $8 \%$ of those with severe paresis (MRC grade < 4) and in $11 \%$ of those with mild paresis (Fig. 2). Among only those cases with preoperative motor deficits, the motor deficit had occurred within
4 weeks prior to the nTMS examination in two-thirds of the patients, whereas the symptoms had already persisted for more than 4 weeks before functional diagnostics were initiated in one-third of the patients. The patients with a shorter case history ( $<4$ weeks) had a lower risk for worsening motor function (15\% vs $40 \%$ in patients with a 4 - to 12 -week case history and $67 \%$ in patients with $>12$-week case history) and better chances of recovery (23\% vs $20 \%$ in patients with a 4 - to 12 -week case history and $0 \%$ in patients with $>12$-week case history) after 3 months ( $\mathrm{p}=$ 0.042).

Patients with a higher KPS score preoperatively had better motor function postoperatively (7 days: Spearman's $\rho=0.318, p=0.001 ; 3$ months: Spearman's $\rho=0.274, p=$ 0.010 ; Table 1).

There was no significant difference between high- and low-grade gliomas in terms of postoperative change in motor status (7 days: $\mathrm{p}=0.487 ; 3$ months: $\mathrm{p}=0.449$ ) and extent of resection (STR vs GTR, $\mathrm{p}=0.190$ ). 


\section{Navigated TMS Mapping}

Bihemispheric motor mapping with nTMS was successfully performed in all patients. No adverse events associated with the examination occurred. Two patients (2\%) complained about a transient tolerable headache. The maximal navigational error, calculated by the system's software after coregistration in each case, was always lower than or equal to $2 \mathrm{~mm}$. The mean RMT for the affected hemisphere was 78.3 V/m (SD 32.5, range 35-293 $\mathrm{V} / \mathrm{m}$ ) and for the healthy hemisphere was $74.9 \mathrm{~V} / \mathrm{m}$ (SD 29.5 , range $29-300 \mathrm{~V} / \mathrm{m}$ ). No significant interhemispheric differences could be found with respect to MEP latency (affected hemisphere [in msec]: mean 23.3, SD 1.9, range 19.1-29.9; healthy hemisphere [in msec]: mean 23.3, SD 1.9 , range $19.0-30.4 ; \mathrm{p}=0.891$ ) or MEP amplitude (affected hemisphere [in $\mu \mathrm{V}$ ]: median 371, SD 1690, range 73-9544; healthy hemisphere [in $\mu \mathrm{V}$ ]: median 403, SD 1387 , range 64-6255; $p=0.248$; data not shown).

\section{Neurophysiological Measurements: RMT Ratio}

Thirty-eight patients (34\%) had an RMT ratio (RMT of the affected hemisphere divided by RMT of the healthy hemisphere) between $90 \%$ and $110 \%$, whereas 36 patients (32\%) had a ratio lower than $90 \%$ and 39 patients (35\%) had a ratio higher than $110 \%$.

The distribution of the RMT ratio according to the motor outcome after 7 days and after 3 months is visualized in Fig. 3.

Twenty-eight percent of patients with a preoperative motor deficit and an RMT ratio $\leq 110 \%$ demonstrated functional improvement at the 3-month follow-up, whereas none of the patients with a preoperative deficit and an RMT ratio $>110 \%$ could regain function (Table 2). Developing a new deficit or experiencing a deterioration in an existing deficit was associated with an RMT ratio $>110 \%$ ( $31 \%$ after 7 days and $33 \%$ after 3 months) versus an RMT ratio $<90 \%$ (19\% after 7 days and 23\% after 3 months) or between $90 \%$ and $110 \%$ (11\% after 7 days and $29 \%$ after 3 months; 7 days: $p=0.031$; 3 months: $p=0.227$; Fig. 3 ).

We found no significant correlation between the RMT of the tumorous hemisphere and the preoperative motor status (paresis group [in V/m]: mean 82.6, SD 39.8, range 36-293; no paresis group [in V/m]: mean 74.5, SD 24.0, range $35-151 ; \mathrm{p}=0.350$ ).

The MEP latency and MEP amplitude values were not significantly associated with the postoperative motor status or outcome.

\section{Navigated TMS-Based Fiber Tracking and Tumor Localization}

Navigated TMS-based fiber tracking was successful in all 54 cases (48\%) in which DTI-capable MRI sequences were acquired. The mean FAT value was 0.26 (SD 0.09, range $0.07-0.48$ ).

According to the nTMS mapping and fiber tracking results, the primary motor cortex and/or the CST were infiltrated (M1) in 21 patients (39\%), and the tumor was $\leq$ $8 \mathrm{~mm}$ from the CST (IntCaps) in 17 patients (31\%). In 16 cases $(30 \%)$, the tumor was $>8 \mathrm{~mm}$ from the CST; in 6 cases (11\%) the tumor was directly adjacent to M1 (M2),
TABLE 2. Predictive modeling

\begin{tabular}{|c|c|}
\hline $\begin{array}{l}\text { Type of } \\
\text { Finding }\end{array}$ & Description \\
\hline Main & $\begin{array}{l}\text { High-risk criteria (presence of any of the following cri- } \\
\text { teria is significantly associated w/ occurrence of new } \\
\text { postop motor deficit, } p=0.032 \text { ) } \\
\text { 1. tumorous infiltration of motor cortex \&/or CST } \\
\text { 2. } \leq 8 \text {-mm distance btwn tumor \& CST } \\
\text { 3. interhemispheric RMT ratio }<90 \% \text { or }>110 \%\end{array}$ \\
\hline Noteworthy & $\begin{array}{l}\text { 1. } 46 \% \text { of patients } w / \geq 1 \text { high-risk criterion \& GTR devel- } \\
\text { oped a new deficit compared to } 7 \% \text { of those w/ STR } \\
\text { 2. } 24 \% \text { of patients w/ preop paresis \& an RMT ratio btwn } \\
90 \% \text { \& } 110 \% \text { demonstrated improvement in motor } \\
\text { function postoperatively vs } 7 \% \text { of those } w / \text { an RMT } \\
\text { ratio }<90 \% \text {; functional improvement never observed in } \\
\text { cases } w / \text { an RMT ratio }>110 \%\end{array}$ \\
\hline
\end{tabular}

and in 10 cases $(18 \%)$ the tumor neither infiltrated nor was directly adjacent to the primary motor cortex (M0). Whereas a critical tumor location (M1 and IntCaps) was responsible for all postoperative motor deteriorations, no functional deterioration due to subcortical injury was observed in any patient with tumors $>8 \mathrm{~mm}$ from the CST (Fig. 2C and 2D; 7 days: $\mathrm{p}=0.014 ; 3$ months: $\mathrm{p}=0.002$ ). Therefore, a distance $\leq 8 \mathrm{~mm}$ was used as the limiting value for further statistical regression analysis.

A greater distance was also associated with a better postoperative muscle strength grade (7 days: median MRC Grade $4.0[\leq 8 \mathrm{~mm}=\mathrm{M} 1$ and IntCaps] vs $5.0[>8 \mathrm{~mm}=$ $\mathrm{M} 2$ and M0], $\mathrm{p}=0.007 ; 3$ months: median MRC $4.0[\leq 8$ $\mathrm{mm}]$ vs $4.5[>8 \mathrm{~mm}], \mathrm{p}=0.078)$.

Moreover, a critical tumor location (M1 vs IntCaps vs M2 vs M0) was associated with a higher rate of STR (56\% vs $20 \%$ vs $67 \%$ vs $0 \%$, respectively) or a lower rate of GTR ( $44 \%$ vs $80 \%$ vs $33 \%$ vs $100 \%$, respectively; $p=0.004$ ).

\section{Extent of Resection and Functional Outcome}

According to postoperative MRI (108 patients [96\%]), GTR was achieved in 54 patients (50\%). In 36 patients $(33 \%)$ residual tumor was detected at volumes smaller than $10 \mathrm{~cm}^{3}$ (STR). A PR (residue $>10 \mathrm{~cm}^{3}$ ) was achieved in 13 cases (12\%), and a biopsy was performed in 5 cases $(5 \%)$.

Patient motor function never changed after a biopsy. Furthermore, we did not observe any improvement in motor status after a patient underwent a PR, and 5 patients in this group (38\%) deteriorated postoperatively. However, there was no significant association between extent of tumor resection and postoperative motor status in general. A subgroup analysis of our high-risk cases (see definition below) revealed that just 1 patient $(7 \%)$ who underwent STR experienced a worsening condition postoperatively, whereas 11 patients (46\%) in the GTR group experienced deterioration ( 7 days: $\mathrm{p}=0.024$; Table 2 ).

\section{Multiple Ordinal Logistic Regression}

In multiple ordinal logistic regression analyses, the pre- 

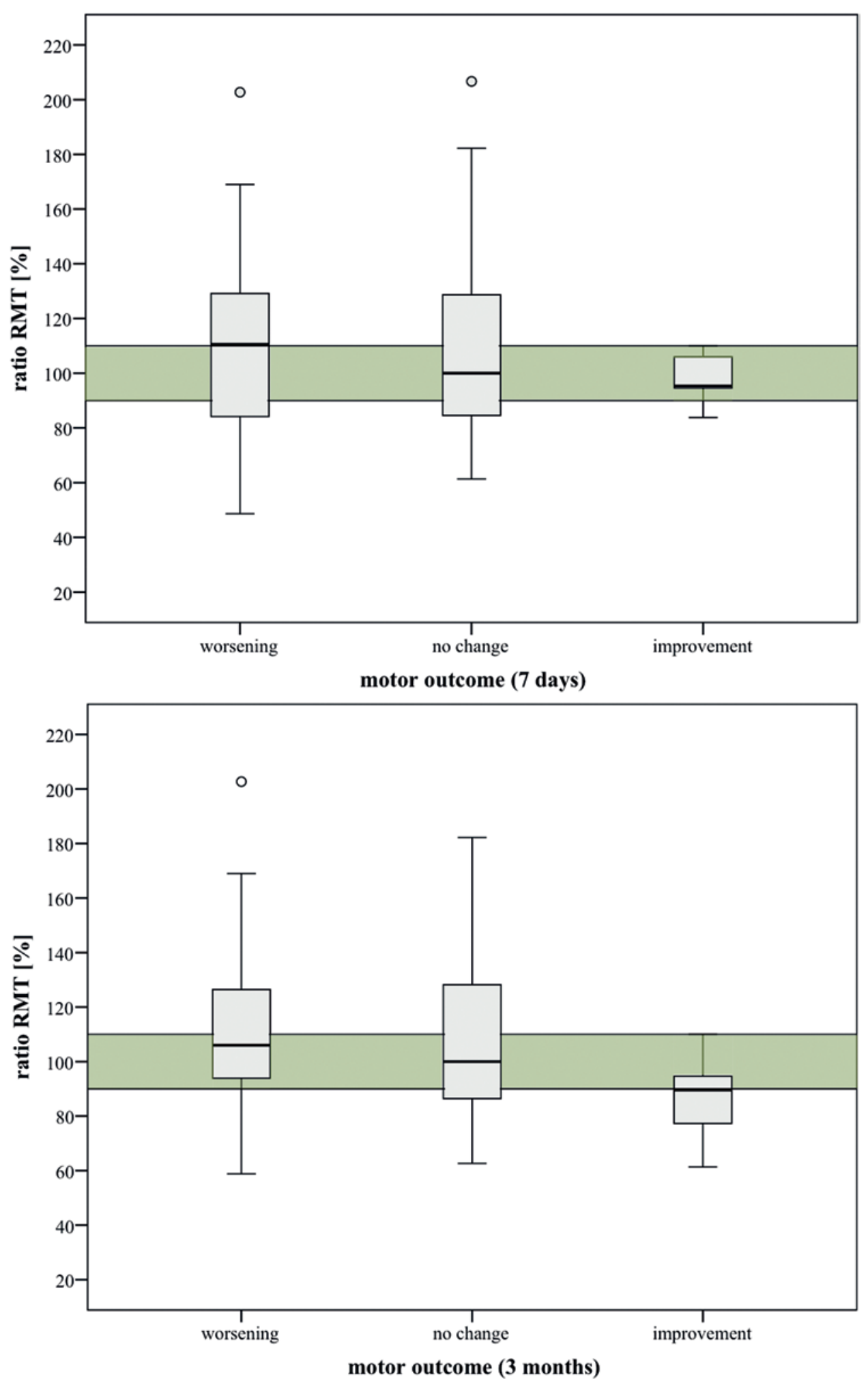

FIG. 3. Box plots illustrating the distribution of the RMT ratio (quotient of RMT of the affected and healthy hemisphere), according to the motor outcome after 7 days (upper) and after 3 months (lower). Physiologically, the RMT does not show a significant interhemispheric difference in healthy subjects. ${ }^{3,9,28,34}$ Box plot shows that most of the patients who could improve had a ratio in the green area (ratio between $90 \%$ and $110 \%$ ). In contrast, cases with postoperative motor deterioration more often had a ratio higher than $110 \%$ (or lower than $90 \%$, respectively). Figure is available in color online only. 
TABLE 3. Multiple ordinal logistic regression analysis of factors significantly associated with the postoperative motor change*

\begin{tabular}{|c|c|c|c|c|c|c|}
\hline \multirow[b]{2}{*}{ Variable } & \multicolumn{3}{|c|}{$\begin{array}{l}\text { Model for Motor Change at } 7 \text { Days } \\
\text { Postoperatively } \dagger\end{array}$} & \multicolumn{3}{|c|}{$\begin{array}{c}\text { Model for Motor Change at } 3 \text { Mos } \\
\text { Postoperatively } \dagger\end{array}$} \\
\hline & OR & $95 \% \mathrm{Cl}$ & p Value & OR & $95 \% \mathrm{Cl}$ & $\mathrm{p}$ Value \\
\hline \multicolumn{7}{|l|}{ Preop motor status } \\
\hline $\mathrm{MRC}$ grade $\leq 3$ & 0.35 & $0.05-2.72$ & 0.318 & 0.03 & $0.00-0.38$ & 0.007 \\
\hline MRC grade 4 & 0.22 & $0.04-1.15$ & 0.073 & 0.72 & $0.17-3.00$ & 0.653 \\
\hline MRC grade 5 & 1 & & & 1 & & \\
\hline \multicolumn{7}{|l|}{ RMT ratio } \\
\hline $90 \%-110 \%$ & 1 & & & - & - & - \\
\hline$>110 \%$ & 13.11 & $2.05-83.71$ & 0.007 & - & - & - \\
\hline$<90 \%$ & 2.71 & $0.51-14.32$ & 0.240 & - & - & - \\
\hline \multicolumn{7}{|l|}{ nTMS-based tumor localization } \\
\hline M1 & 18.81 & $2.32-152.76$ & 0.006 & 9.05 & $1.05-78.27$ & 0.045 \\
\hline IntCaps & 22.54 & $2.59-196.42$ & 0.005 & 7.62 & $0.86-67.72$ & 0.068 \\
\hline M2 & 4.73 & $0.32-70.39$ & 0.259 & 1.14 & $0.08-15.80$ & 0.920 \\
\hline M0 & 1 & & & 1 & & \\
\hline \multicolumn{7}{|l|}{ Model fit } \\
\hline$R^{2}$ (pseudo) & 0.30 & & & 0.19 & & \\
\hline No. (\%) correctly classified & $43(79.6 \%)$ & & & $26(61.9 \%)$ & & \\
\hline
\end{tabular}

operative motor status, RMT ratio, and nTMS-based tumor localization remained significantly associated with the postoperative motor change at 7 days after surgery $\left(\mathrm{R}^{2}=\right.$ 0.30 ; Table 3). A pathological RMT ratio $(>110 \%$ and $<$ 90\%) was associated with a lower probability of improvement and a higher probability of worsening, as compared with an RMT ratio between $90 \%$ and $110 \%$. The same was true for critical tumor localizations.

When analyzing the postoperative motor change after 3 months, the RMT ratio was not significantly associated $\left(\mathrm{R}^{2}=0.19\right.$; Table 3). As in the model for the 7-day motor status change, a lower preoperative motor status and safer tumor localizations were associated with a lower probability of worsening.

\section{Risk Stratification}

Using results of the logistic regression analysis, we classified patients into high- and low-risk groups (for postoperative deterioration of motor function). High-risk criteria were glioma infiltrating the primary motor cortex (M1) and/or a distance $\leq 8 \mathrm{~mm}$ between tumor and CST and/ or a pathological excitability of the motor cortices (interhemispheric RMT ratio $<90 \%$ or $>110 \%$ ). On the other hand, we could assign patients with a noneloquently located tumor (M0, M2, distance to CST $>8 \mathrm{~mm}$ ) with 2 equally excitable motor cortices into a group of low-risk cases (Table 2). Thus, there were $46(85 \%)$ high-risk cases and $8(15 \%)$ low-risk cases.

Regarding motor outcome, the likelihood of motor improvement was higher in the low-risk group (7 days: $25 \%$;
3 months: 13\%) than in the high-risk group (7 days: $4 \%$; 3 months: $9 \%$ ). None of the patients in the low-risk group and 13 patients (38\%) in the high-risk group developed a new permanent deficit ( 7 days: $p=0.032 ; 3$ months: $p=0.106$ ).

The individual probability for the postoperative motor outcome after 7 days and 3 months can be calculated using the equations in Table 4.

\section{Discussion}

\section{Main Study Finding}

Functional-anatomical and neurophysiological measurements derived from presurgical nTMS analysis allowed us, for the first time, to assess the risk of functional deterioration and the potential for functional recovery in rolandic glioma surgery by using objective data. The most relevant results for presurgically balancing risks and benefits, counseling patients, and planning surgeries are summarized in Table 2.

\section{Background}

For gliomas it has been shown that residual tumor volume directly correlates with progression-free survival. ${ }^{1,5,12,31}$ Therefore, in most cases the surgical goal is to achieve as extensive a resection of the tumor as is "safely" possible. However, brain tumor surgery in eloquent regions always includes the risk of inducing a new functional deficit, which decreases the patient's quality of life and correlates with shorter survival. ${ }^{21}$

Recent studies have demonstrated that preoperative 
TABLE 4. Equations for the individual probability for postoperative motor outcome

\begin{tabular}{|c|c|}
\hline Outcome & Equation \\
\hline $\begin{array}{l}\text { After } 7 \\
\text { days }\end{array}$ & 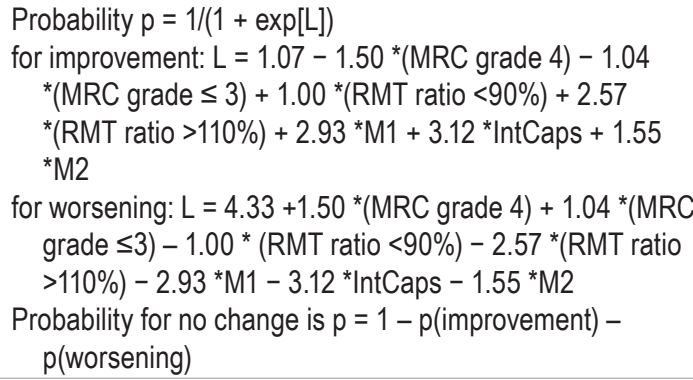 \\
\hline $\begin{array}{l}\text { After } 3 \\
\text { mos }\end{array}$ & 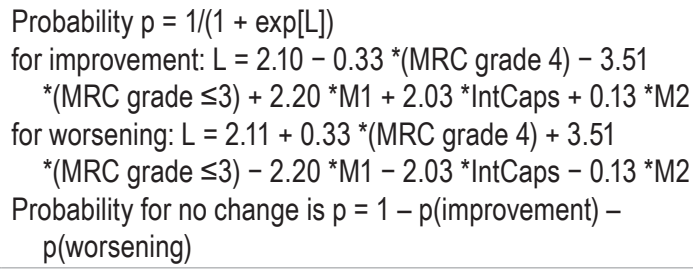 \\
\hline Example & 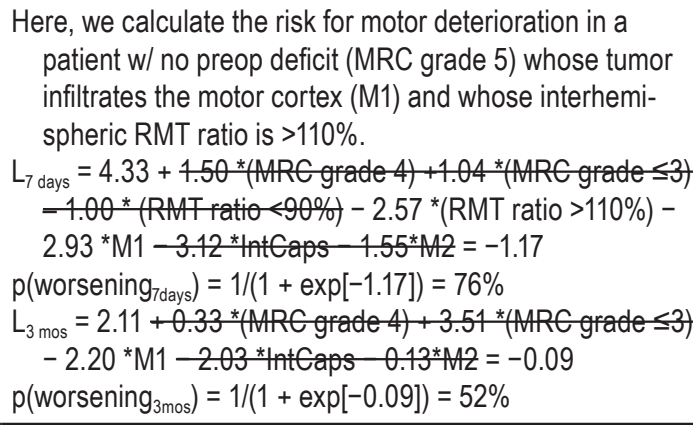 \\
\hline
\end{tabular}

$\exp (\mathrm{L})=\mathrm{e}^{\mathrm{L}}$, where $\mathrm{e}$ is Euler's number.

* The value in front of the asterisk should be applied when the condition behind the asterisk is fulfilled. In the example, the portion of the equation with a line through it signifies the portion that does not apply.

nTMS mapping in patients with brain tumor in a presumed motor eloquent location leads to a higher rate of GTR and longer progression-free survival while at the same time reducing the rate of permanent deficits. ${ }^{10,16}$ In addition, it has been shown that the insertion of nTMS data into CST tractography increases the accuracy and specificity of fiber tracking in a user-independent manner compared with those achieved with conventional fiber tracking based on anatomical landmarks. ${ }^{8,11,15}$ To further improve the clinical utility of nTMS, we propose a model of nTMS-based risk stratification. The model allows one to identify cases at high risk for incurring a new postoperative motor deficit by using objective measures. In addition, the model predicts which cases with preexisting deficits have a chance of recovery. The nTMS analysis facilitates preoperative risk-benefit balancing and patient counseling as well as the consequent decision making.

\section{Risk Stratification Based on nTMS}

On the cortical level, tumorous invasion of the primary motor cortex - that is, nTMS-proven function within the border zone of the cortical aspect of the glioma-is a risk factor for postoperative functional deficit. Subcortically, nTMS-based fiber tracking has recently been introduced as a reliable method for visualizing the $\mathrm{CST}^{6,8,33}$ In the current study, no new postoperative motor deficit was observed when the minimum distance between tumor and CST was $>8 \mathrm{~mm}$. If there is no other risk factor, such cases can be categorized into the low-risk group and GTR should be the surgical goal. None of the patients in the lowrisk group had a deteriorated motor status postoperatively.

Careful surgical planning and treatment are indicated in high-risk cases, in which the minimum distance between tumor and CST is $\leq 8 \mathrm{~mm}$. On one hand, patients with direct tumor infiltration of the CST had the highest rate of motor worsening. On the other hand, we also observed cases with postoperative improvement in motor function, which probably reflects a more conservative surgical strategy in this group resulting in (transient) functional improvement through tumor debulking. In this context, the high rate of GTR-associated (46\%) deficits in our subgroup analysis of high-risk cases is a very important finding. The fact that just 1 patient with STR experienced deterioration may reinforce the necessity of carefully balancing the additional benefit of GTR against the significant risk of inducing a permanent deficit. The use of IOM must be seen as mandatory in high-risk cases.

With respect to cortical excitability, equally excitable hemispheres (RMT ratio 90\%-110\%), as occur in healthy subjects, $3,9,28,34$ were associated with a better outcome and can therefore be handled as low-risk cases. In contrast, patients with a pathological RMT ratio should be treated as high-risk cases. Although the RMT ratio was only included in the model for motor outcome after 7 days, the results also indicated a correlation with long-term deficits. However, a patient's (motor) status on Day 7 is a relevant factor since it affects the timing of further treatment, for example, adjuvant therapy. At this moment there is no proven explanation for this correlation. Perez and Cohen, among others, reported a significant imbalance in interhemispheric inhibition in patients with paresis after stroke. $^{22}$ More specifically, a strong inhibition of the affected hemisphere by the healthy one leads to increased motor deficits and reduced capacity for functional rehabilitation. Our results fit this observation since the rate of motor deterioration was especially high in patients with an RMT ratio $>110 \%$, that is, with impaired excitability of the tumor hemisphere. Moreover, patients with impaired excitability of the tumorous hemisphere and a preexisting motor deficit never showed improvement in motor function postoperatively.

In addition to the nTMS-based factors, the preoperative motor status and the duration of motor symptoms are also significantly associated with the relative motor outcome. Regarding clinical status, patients with a preoperative deficit and a shorter case history had a better chance of improving their motor status.

Our multiple ordinal logistic regression analysis allowed us to classify tumors in motor-associated areas into high-risk and low-risk cases. This nTMS-based risk stratification enables objective and individualized risk-benefit balancing and facilitates patient counseling and surgical planning. 


\section{Study Limitations}

The proposed nTMS-based risk stratification model is based on statistically highly significant data. Yet, we cannot exclude the possibility that our results were influenced by the specific decision-making processes and treatment algorithms of our department. This study is the first to disclose a predictive utility of nTMS. Next, the presented results must be validated by external data.

\section{Conclusions}

The nTMS-based risk stratification can be routinely applied when treating patients with a brain tumor in presumed motor eloquent regions. In high-risk cases the use of IOM is strongly advised, whereas IOM in low-risk cases is not obligatory. In addition, the patient can now be better informed about individual chances and risks of tumor resection.

\section{References}

1. Almenawer SA, Badhiwala JH, Alhazzani W, Greenspoon J, Farrokhyar F, Yarascavitch B, et al: Biopsy versus partial versus gross total resection in older patients with high-grade glioma: a systematic review and meta-analysis. Neuro Oncol 17:868-881, 2015

2. Barker AT, Jalinous R, Freeston IL: Non-invasive magnetic stimulation of human motor cortex. Lancet 325:1106-1107, 1985

3. Bashir S, Perez JM, Horvath JC, Pena-Gomez C, Vernet M, Capia A, et al: Differential effects of motor cortical excitability and plasticity in young and old individuals: a transcranial magnetic stimulation (TMS) study. Front Aging Neurosci 6:111, 2014

4. Berger MS, Deliganis AV, Dobbins J, Keles GE: The effect of extent of resection on recurrence in patients with low grade cerebral hemisphere gliomas. Cancer 74:1784-1791, 1994

5. Capelle L, Fontaine D, Mandonnet E, Taillandier L, Golmard JL, Bauchet L, et al: Spontaneous and therapeutic prognostic factors in adult hemispheric World Health Organization Grade II gliomas: a series of 1097 cases: clinical article. J Neurosurg 118:1157-1168, 2013

6. Conti A, Raffa G, Granata F, Rizzo V, Germanò A, Tomasello F: Navigated transcranial magnetic stimulation for "somatotopic" tractography of the corticospinal tract. Neurosurgery 10 (Suppl 4):542-554, 2014

7. Duffau H, Lopes M, Arthuis F, Bitar A, Sichez JP, Van Effenterre R, et al: Contribution of intraoperative electrical stimulations in surgery of low grade gliomas: a comparative study between two series without (1985-96) and with (19962003) functional mapping in the same institution. J Neurol Neurosurg Psychiatry 76:845-851, 2005

8. Forster MT, Hoecker AC, Kang JS, Quick J, Seifert V, Hattingen E, et al: Does navigated transcranial stimulation increase the accuracy of tractography? A prospective clinical trial based on intraoperative motor evoked potential monitoring during deep brain stimulation. Neurosurgery 76:766-776, 2015

9. Forster MT, Limbart M, Seifert V, Senft C: Test-retest reliability of navigated transcranial magnetic stimulation of the motor cortex. Neurosurgery 10 (Suppl 1):51-56, 2014

10. Frey D, Schilt S, Strack V, Zdunczyk A, Rösler J, Niraula B, et al: Navigated transcranial magnetic stimulation improves the treatment outcome in patients with brain tumors in motor eloquent locations. Neuro Oncol 16:1365-1372, 2014

11. Frey D, Strack V, Wiener E, Jussen D, Vajkoczy P, Picht T:
A new approach for corticospinal tract reconstruction based on navigated transcranial stimulation and standardized fractional anisotropy values. Neuroimage 62:1600-1609, 2012

12. Jakola AS, Myrmel KS, Kloster R, Torp SH, Lindal S, Unsgård G, et al: Comparison of a strategy favoring early surgical resection vs a strategy favoring watchful waiting in low-grade gliomas. JAMA 308:1881-1888, 2012

13. Kombos T, Picht T, Derdilopoulos A, Suess O: Impact of intraoperative neurophysiological monitoring on surgery of high-grade gliomas. J Clin Neurophysiol 26:422-425, 2009

14. Kombos T, Süss O, Vajkoczy P: Subcortical mapping and monitoring during insular tumor surgery. Neurosurg Focus 27(4):E5, 2009

15. Krieg SM, Buchmann NH, Gempt J, Shiban E, Meyer B, Ringel F: Diffusion tensor imaging fiber tracking using navigated brain stimulation-a feasibility study. Acta Neurochir (Wien) 154:555-563, 2012

16. Krieg SM, Sabih J, Bulubasova L, Obermueller T, Negwer C, Janssen I, et al: Preoperative motor mapping by navigated transcranial magnetic brain stimulation improves outcome for motor eloquent lesions. Neuro Oncol 16:1274-1282, 2014

17. Krieg SM, Shiban E, Buchmann N, Gempt J, Foerschler A, Meyer B, et al: Utility of presurgical navigated transcranial magnetic brain stimulation for the resection of tumors in eloquent motor areas. J Neurosurg 116:994-1001, 2012

18. Lee CH, Kim DG, Kim JW, Han JH, Kim YH, Park CK, et al: The role of surgical resection in the management of brain metastasis: a 17-year longitudinal study. Acta Neurochir (Wien) 155:389-397, 2013

19. Louis DN, Ohgaki H, Wiestler OD, Cavenee WK, Burger PC, Jouvet A, et al: The 2007 WHO classification of tumours of the central nervous system. Acta Neuropathol 114:97-109, 2007

20. Medical Research Council: Aids to the Examination of the Peripheral Nervous System. Memorandum No. 45. London: Her Majesty's Stationery Office, 1981

21. McGirt MJ, Mukherjee D, Chaichana KL, Than KD, Weingart JD, Quiñones-Hinojosa A: Association of surgically acquired motor and language deficits on overall survival after resection of glioblastoma multiforme. Neurosurgery 65:463-470, 2009

22. Perez MA, Cohen LG: The corticospinal system and transcranial magnetic stimulation in stroke. Top Stroke Rehabil 16:254-269, 2009

23. Picht T, Mularski S, Kuehn B, Vajkoczy P, Kombos T, Suess O: Navigated transcranial magnetic stimulation for preoperative functional diagnostics in brain tumor surgery. Neurosurgery 65 (6 Suppl):93-99, 2009

24. Picht T, Schmidt S, Brandt S, Frey D, Hannula H, Neuvonen T, et al: Preoperative functional mapping for rolandic brain tumor surgery: comparison of navigated transcranial magnetic stimulation to direct cortical stimulation. Neurosurgery 69:581-588, 2011

25. Picht T, Schulz J, Hanna M, Schmidt S, Suess O, Vajkoczy P: Assessment of the influence of navigated transcranial magnetic stimulation on surgical planning for tumors in or near the motor cortex. Neurosurgery 70:1248-1257, 2012

26. Picht T, Strack V, Schulz J, Zdunczyk A, Frey D, Schmidt S, et al: Assessing the functional status of the motor system in brain tumor patients using transcranial magnetic stimulation. Acta Neurochir (Wien) 154:2075-2081, 2012

27. Rossini PM, Barker AT, Berardelli A, Caramia MD, Caruso G, Cracco RQ, et al: Non-invasive electrical and magnetic stimulation of the brain, spinal cord and roots: basic principles and procedures for routine clinical application. Report of an IFCN committee. Electroencephalogr Clin Neurophysiol 91:79-92, 1994

28. Säisänen L, Julkunen P, Niskanen E, Hukkanen T, Mervaala 
E, Karhu J, et al: Short- and intermediate-interval cortical inhibition and facilitation assessed by navigated transcranial magnetic stimulation. J Neurosci Methods 195:241-248, 2011

29. Sanai N, Berger MS: Glioma extent of resection and its impact on patient outcome. Neurosurgery 62:753-764, 264-266, 2008

30. Schag CC, Heinrich RL, Ganz PA: Karnofsky performance status revisited: reliability, validity, and guidelines. J Clin Oncol 2:187-193, 1984

31. Stummer W, Reulen HJ, Meinel T, Pichlmeier U, Schumacher W, Tonn JC, et al: Extent of resection and survival in glioblastoma multiforme: identification of and adjustment for bias. Neurosurgery 62:564-576, 2008

32. Tarapore PE, Tate MC, Findlay AM, Honma SM, Mizuiri D, Berger MS, et al: Preoperative multimodal motor mapping: a comparison of magnetoencephalography imaging, navigated transcranial magnetic stimulation, and direct cortical stimulation. J Neurosurg 117:354-362, 2012

33. Weiss C, Tursunova I, Neuschmelting V, Lockau H, Nettekoven C, Oros-Peusquens AM, et al: Improved nTMSand DTI-derived CST tractography through anatomical ROI seeding on anterior pontine level compared to internal capsule. Neuroimage Clin 7:424-437, 2015

34. Zdunczyk A, Fleischmann R, Schulz J, Vajkoczy P, Picht T: The reliability of topographic measurements from navigated transcranial magnetic stimulation in healthy volunteers and tumor patients. Acta Neurochir (Wien) 155:1309-1317, 2013

\section{Disclosures}

The authors report no conflict of interest concerning the materials or methods used in this study or the findings specified in this paper.

\section{Author Contributions}

Conception and design: Rosenstock, Acker, Picht. Acquisition of data: Rosenstock, Schwarzer, Kulchytska. Analysis and interpretation of data: Rosenstock, Picht. Drafting the article: Rosenstock. Critically revising the article: Rosenstock, Grittner, Acker, Vajkoczy, Picht. Reviewed submitted version of manuscript: all authors. Approved the final version of the manuscript on behalf of all authors: Rosenstock. Statistical analysis: Rosenstock, Grittner. Administrative/technical/material support: Rosenstock, Schwarzer, Kulchytska, Vajkoczy, Picht. Study supervision: Vajkoczy, Picht.

\section{Correspondence}

Tizian Rosenstock, Department of Neurosurgery, Charité University Medicine, Augustenburger Platz 1, Berlin 13353, Germany. email: tizian.rosenstock@charite.de. 